\title{
A conserved Bach current
}

\author{
E N Glass ${ }^{1}$ \\ Department of Physics, University of Michigan, Ann Arbor, MI 48109, USA
}

Received 11 June 2001

Published 5 September 2001

Online at stacks.iop.org/CQG/18/3935

\begin{abstract}
The Bach tensor and a vector which generates conformal symmetries allow a conserved 4-current to be defined. The Bach 4-current gives rise to a quasilocal 2-surface expression for power per luminosity distance in the Vaidya exterior of collapsing fluid interiors. This is interpreted in terms of entropy generation.
\end{abstract}

PACS numbers: 0420J, 0440N

\section{Introduction}

Gravitational theory allows a conserved current $J_{\text {EIN }}^{\alpha}$ to be formed from the Einstein tensor with a symmetry generated by Killing vector $k^{\beta}$ :

$$
J_{\mathrm{EIN}}^{\alpha}=G^{\alpha}{ }_{\beta} k^{\beta} .
$$

The current $J_{\mathrm{EIN}}^{\alpha}$ is conserved since the Einstein tensor is divergence-free and $k^{\beta}$ satisfies Killing's equation. In a similar fashion we define

$$
J_{\mathrm{BACH}}^{\alpha}:=B^{\alpha}{ }_{\beta} \xi^{\beta}
$$

where $B_{\alpha \beta}$ is the Bach tensor, a symmetric, divergence-free, trace-free tensor [1-4]. $\xi^{\beta}$ is a generator of conformal maps which include Killing symmetry and homothety as special cases. $J_{\mathrm{BACH}}^{\alpha}$ is conserved because the Bach tensor is divergence-free and the symmetrized covariant derivative of the conformal generator $\xi^{\beta}$ is $\xi_{(\alpha ; \beta)}=2 \Psi\left(x^{v}\right) g_{\alpha \beta}$. Since the Bach tensor is trace-free it follows that $B_{\alpha \beta} \xi^{(\alpha ; \beta)}$ vanishes.

The Bach tensor is a second derivative of the Weyl tensor $C_{\alpha \mu \beta \nu}$ plus a Ricci tensor term

$$
B_{\alpha \beta}=\nabla^{\mu} \nabla^{\nu} C_{\alpha \mu \beta \nu}+\frac{1}{2} R^{\mu \nu} C_{\alpha \mu \beta \nu} .
$$

For metrics with non-zero Bach tensor, we can think of $B_{\alpha \beta}(g)=\kappa_{\text {weyl }} S_{\alpha \beta}$ as the field equations of conformal Weyl gravity [5], with symmetric $S_{\alpha \beta}$ analogous to $T_{\alpha \beta}$ of Einstein's theory.

There exist superpotentials for $J_{\text {EIN }}^{\alpha}$ which lead to an Einstein mass by integrating the superpotential over 2-surface $S^{2}$. Three of the superpotentials do not require a coordinate

1 Permanent address: Physics Department, University of Windsor, Ontario N9B 3P4, Canada. 
choice: the Komar potential [6], the Penrose-Goldberg potential [7] and the Taub potential [8]. However, each of those three superpotentials is restricted to special systems. For more general potentials derived from the Hilbert action and pseudotensors, the Einstein energy is known to be ambiguously defined because it depends on the choice of background coordinates. Nevertheless, one may use the 2-surface construction to define sensible notions of total gravitational energy. Indeed, for asymptotic flatness, say, towards future null infinity $\mathcal{I}^{+}$, $S^{2}$ tends to a 'nice' cut of $\mathcal{I}^{+}$, a round 2 -sphere. The (suitably unique) choice of asymptotically Cartesian coordinates ensures that the Einstein energy agrees with the accepted BondiSachs notion of total energy [9] within some quasilocal (i.e. finite) 2-surface $S^{2}$. One of the first fundamental expositions of quasilocal mass (and angular momentum) was given by Penrose [10]. A more recent and well known treatment of quasilocal energy is the work of Brown et al [11].

In this work we introduce the conserved Bach current as a tool, in general relativity, to study collapsing star models. In particular, we use the properties of the Bach tensor and Bach current to study collapsing spherical fluid interiors matched to a Vaidya exterior and analyse the power flow. $\xi^{\alpha}$ generates a matter symmetry in the Vaidya exterior, weaker than conformal symmetry, but $\xi_{(\alpha ; \beta)}$ annihilates the Bach tensor and so the current is conserved. The volume integral of $J_{\mathrm{BACH}}^{\alpha}$ leads to a 2-surface integral which provides a quasilocal value for the Vaidya power gradient. Covariant thermodynamics of irreversible systems is used to interpret the Bach current for the Vaidya exterior. The Vaidya power gradient is related to the rate of entropy increase and the number of states the energy resides in.

We can view a star model as a single state drawn from a microcanonical ensemble. The initial values of all the observables are at their equilibrium values (say, all zero), then, as a function of time, they will not remain exactly zero but will execute small fluctuations about their zero equilibrium values. The Vaidya exterior dissipates energy by sending 'short-wavelength photons' to null infinity. It is the Bach current and the rate of entropy increase that allows one, via the fluctuation-dissipation theorem, to measure the rate at which a deviation from equilibrium decays.

\section{Conventions and notation}

In this work Greek indices range over $(0,1,2,3)=(u, r, \vartheta, \varphi)$. Sign conventions are $2 A_{\nu ;[\alpha \beta]}=A_{\mu} R_{\nu \alpha \beta}^{\mu}$, and $R_{\alpha \beta}=R_{\alpha \beta \nu}^{v}$. Overdots abbreviate $\partial / \partial u$ and primes abbreviate $\partial / \partial r$. The metric signature is $(+,-,-,-)$ and the field equations are $G_{\mu \nu}=-8 \pi T_{\mu \nu}$.

\section{Some properties of the Bach tensor}

(a) A variational derivative of the Lagrangian density $\mathcal{L}=\sqrt{-g} C_{\alpha \beta \gamma \delta} C^{\alpha \beta \gamma \delta}$ yields the Bach tensor

$$
\sqrt{-g} B_{\alpha \beta}=\frac{\delta \mathcal{L}}{\delta g^{\alpha \beta}},
$$

and the Lagrangian provides the field equation

$$
B_{\alpha \beta}(g)=0
$$

Solutions of equation (4) are vacuum solutions of conformal Weyl gravity [5]. 
(b) Kozameh et al [2] proved that any solution of equation (4) which is also conformal to $C$ spaces is conformal to an Einstein metric (Einstein metrics have Ricci tensor proportional to the metric). For an example of a metric which satisfies $B_{\alpha \beta}(g)=0$ but is not an Einstein metric see Nurowski and Plebanski [12].

(c) Vacuum spacetimes, conformally flat spacetimes, and Einstein spacetimes have vanishing Bach tensor.

(d) For $P_{\mu \nu}=R_{\mu \nu}-(R / 6) g_{\mu \nu}$, the Bianchi identities allow the alternate expression

$$
B_{\alpha \beta}=\nabla^{\mu} \nabla_{[\alpha} P_{\mu] \beta}+\frac{1}{2} P^{\mu v} C_{\alpha \mu \beta \nu} .
$$

(e) Under conformal map $\hat{g}_{\alpha \beta}=\Omega^{2} g_{\alpha \beta}$, the Bach tensor transforms as $\hat{B}_{\alpha \beta}=B_{\alpha \beta}$.

\section{The Vaidya spacetime}

\subsection{Vaidya interiors}

The first Vaidya interior was constructed by Synge [13]. In that work an incoherent shell of radiation is joined to Vaidya. Santos [14] joined a collapsing fluid with radial heat flow to a Vaidya exterior. Glass [15] studied a two-fluid interior, a collapsing perfect fluid with an outgoing null fluid matched to a Vaidya exterior. Continuity of the first and second fundamental forms expressed conservation of momentum across the boundary and revealed the boundary to be the perfect fluid zero-pressure surface. This is the interior we have in mind to model the energy flow considered here. As the perfect fluid collapses, energy is removed by the null fluid. In the Vaidya exterior, the outgoing short-wavelength photons carry energy to future null infinity.

\subsection{Vaidya exterior}

The Vaidya metric in outgoing null coordinates, with arbitrary mass function $m(u)$ and $A:=1-2 m(u) / r$, is given by

$$
g_{\alpha \beta}^{V a d} \mathrm{~d} x^{\alpha} \mathrm{d} x^{\beta}=A \mathrm{~d} u^{2}+2 \mathrm{~d} u \mathrm{~d} r-r^{2}\left(\mathrm{~d} \vartheta^{2}+\sin ^{2} \vartheta \mathrm{d} \varphi^{2}\right) .
$$

We use a Newman-Penrose null tetrad:

$$
\begin{array}{ll}
l^{\alpha} \partial_{\alpha}=\partial_{r}, & l_{\alpha} \mathrm{d} x^{\alpha}=\mathrm{d} u, \\
n^{\alpha} \partial_{\alpha}=\partial_{u}-(A / 2) \partial_{r}, & n_{\alpha} \mathrm{d} x^{\alpha}=\mathrm{d} r+(A / 2) \mathrm{d} u, \\
m^{\alpha} \partial_{\alpha}=\left(r^{-1} / \sqrt{2}\right)\left(\partial_{\vartheta}+\frac{\mathrm{i}}{\sin \vartheta} \partial_{\varphi}\right), & m_{\alpha} \mathrm{d} x^{\alpha}=-(r / \sqrt{2})(\mathrm{d} \vartheta+\mathrm{i} \sin \vartheta \mathrm{d} \varphi),
\end{array}
$$

with non-zero spin coefficients

$$
\begin{aligned}
& \rho=-1 / r, \quad \mu=-A /(2 r), \\
& \alpha=-\cot \vartheta /(2 \sqrt{2} r)=-\beta, \\
& \gamma=A^{\prime} / 4 .
\end{aligned}
$$

The Weyl tensor is given in terms of a basis set of anti self-dual bivectors $\left(\Psi_{2}=-m(u) / r^{3}\right)$

$$
C^{\alpha \mu \beta \nu}=\left[m(u) / r^{3}\right]\left(U^{\alpha \mu} V^{\beta \nu}+V^{\alpha \mu} U^{\beta v}+M^{\alpha \mu} M^{\beta \nu}+\text { c.c. }\right),
$$

where

$U^{\alpha \beta}=2 \bar{m}^{[\alpha} n^{\beta]}, \quad M^{\alpha \beta}=2 l^{[\alpha} n^{\beta]}-2 m^{[\alpha} \bar{m}^{\beta]}, \quad V^{\alpha \beta}=2 l^{[\alpha} m^{\beta]}$. 
The Vaidya spacetime is Petrov type $\boldsymbol{D}$ and has the invariant relation

$$
R_{\alpha \beta \mu \nu} R^{\alpha \beta \mu \nu}=C_{\alpha \beta \mu \nu} C^{\alpha \beta \mu \nu}=48 \Psi_{2}^{2} .
$$

The Ricci and energy-momentum tensors are

$$
R_{\mu \nu}=-8 \pi T_{\mu \nu}=\left(\frac{2 \dot{m}}{r^{2}}\right) l_{\mu} l_{\nu}
$$

The standard interpretation of the Vaidya energy flow was given by Lindquist et al [16] with respect to an observer at rest at future null infinity whose worldline tangent is

$$
\hat{u}^{\mu} \partial_{\mu}=A^{-1 / 2} \partial_{u}=A^{-1 / 2}\left(n^{\mu}+\frac{1}{2} A l^{\mu}\right) \partial_{\mu} .
$$

The local energy flux $f$ is

$$
T_{\mu \nu} \hat{u}^{\mu} \hat{u}^{\nu}=-\left(\frac{\dot{m}}{4 \pi r^{2}}\right) \frac{1}{1-2 m(u) / r},
$$

and the total luminosity seen at infinity is $L_{\infty}(u)=\lim _{r \rightarrow \infty} 4 \pi r^{2} f=-\dot{m}$.

Substitution of the Weyl tensor equation (9) and the Ricci tensor equation (12) into equation (2) yields the Bach tensor

$$
B_{\alpha \beta}\left(g^{\text {Vad }}\right)=\left(\frac{2 \ddot{m}}{r^{3}}\right) l_{\alpha} l_{\beta}+\left(\frac{2 \dot{m}}{r^{4}}\right)\left(l_{\alpha} n_{\beta}+n_{\alpha} l_{\beta}+m_{\alpha} \bar{m}_{\beta}+\bar{m}_{\alpha} m_{\beta}\right) .
$$

The independent eigenvectors of $B_{\alpha \beta}\left(g^{\text {Vad }}\right)$ are $\partial_{r}=l^{\alpha} \partial_{\alpha}, \partial_{\vartheta}=(r / \sqrt{2})\left(m^{\alpha}+\bar{m}^{\alpha}\right) \partial_{\alpha}$, and $\partial_{\varphi}=-\mathrm{i}(r / \sqrt{2}) \sin \vartheta\left(m^{\alpha}-\bar{m}^{\alpha}\right) \partial_{\alpha}$.

\section{Vaidya conserved current}

The generator of time translations is $\partial_{u}=\xi^{\alpha} \partial_{\alpha}$. The time dependence of $m(u)$ prevents $\xi^{\alpha}$ from being a Killing symmetry but $\xi^{\alpha}$ generates a matter symmetry since $\mathcal{L}_{\xi} g_{\alpha \beta}^{\text {Vad }}=-r R_{\alpha \beta}$. The Bach current $J_{\mathrm{BACH}}^{\alpha}=B^{\alpha \beta} \xi_{\beta}$ for metric $g^{\mathrm{Vad}}$ will be labelled $J_{\text {Vad }}^{\alpha}$. The 4-current is conserved since $B_{\alpha \beta} \xi^{(\alpha ; \beta)}=B_{\alpha \beta} l^{\alpha} l^{\beta}=0$. Stronger symmetry can be imposed by restricting the mass function. With a linear mass function, the Vaidya metric has been used to study selfsimilar collapse [17]. This restriction was anticipated by the author [18] for $m(u)=m_{0}+m_{1} u$ and homothetic symmetry $\xi^{\alpha} \partial_{\alpha}=\left(u_{0}+u\right) \partial_{u}+\partial_{r}$ where $\mathcal{L}_{\xi} g_{\alpha \beta}^{\mathrm{Vad}}=2 g_{\alpha \beta}^{\mathrm{Vad}}$.

With $\xi^{\alpha}=(A / 2) l^{\alpha}+n^{\alpha}$, the conserved 4-current is

$$
J_{V a d}^{\alpha}=\left(\frac{2 \dot{m}}{r^{4}}\right) n^{\alpha}+\left(\frac{2 \ddot{m}}{r^{3}}+A \frac{\dot{m}}{r^{4}}\right) l^{\alpha} .
$$

The 4-current is more transparent when written in terms of curve tangents

$$
J_{V a d}^{\alpha} \partial_{\alpha}=\left(\frac{2 \dot{m}}{r^{4}}\right) \partial_{u}+\left(\frac{2 \ddot{m}}{r^{3}}\right) \partial_{r},
$$

and is similar to a conserved Maxwell current $\sqrt{-g} J_{\text {Max }}^{\alpha}=\sqrt{-g}(\rho, \vec{J})$. By analogy we call $2 \dot{m} / r^{2}$ the Bach 'charge density'. Examining $\sqrt{-g} J_{\text {Vad }}^{\alpha}$ tells one that $2 \dot{m} / r^{2}$ is the flow across $u=$ constant null 3 -surfaces and $2 \ddot{m} / r$ is the flow across $r=$ constant timelike 3 -surfaces. This would be equally apparent upon integrating $\partial_{\alpha}\left(\sqrt{-g} J_{\text {Vad }}^{\alpha}\right)=0$ over a 4-volume bounded by four such surfaces. 
A potential exists for $J_{V a d}^{\alpha}$, namely the bivector density

$$
U_{V a d}^{\alpha \beta}=\sqrt{-g}\left(\frac{\dot{m}}{r^{3}}\right) 4 l^{[\alpha} n^{\beta]}
$$

with $\sqrt{-g} J_{\text {Vad }}^{\alpha}=\partial_{\beta} U_{\text {Vad }}^{\alpha \beta}$. We integrate over a $u=$ constant null 3-surface $\mathcal{N}$ :

$$
\int_{\mathcal{N}} \sqrt{-g} J_{V a d}^{\alpha} \mathrm{d} S_{\alpha}=\oint_{\partial \mathcal{N}} U_{V a d}^{\alpha \beta} \mathrm{d} S_{\alpha \beta}
$$

where $\mathrm{d} S_{\alpha}=l_{\alpha} \mathrm{d} r \mathrm{~d} \vartheta \mathrm{d} \varphi$ and $\partial \mathcal{N}$ is a closed 2-surface bounding $\mathcal{N}$ with $\mathrm{d} S_{\alpha \beta}=l_{[\alpha} n_{\beta]} \mathrm{d} \vartheta \mathrm{d} \varphi$. The integral of the Bach 'charge density' over $\partial \mathcal{N}$ provides the quasilocal Bach charge within $\partial \mathcal{N}$, which here is the Vaidya luminosity divided by luminosity distance (and so we call it 'luminosity gradient')

$$
\oint_{\partial \mathcal{N}}\left(\frac{\dot{m}}{r}\right) \sin \vartheta 4 l^{[\alpha} n^{\beta]} \mathrm{d} S_{\alpha \beta}=-8 \pi \frac{\dot{m}}{r},
$$

where $r$ ranges from the spacelike surface $r=2 m$ to future null infinity.

\section{Thermodynamic interpretation}

The Eckart theory of dissipative fluids is the simplest relativistic generalization of NavierStokes theory, but Eckart's theory suffers from a lack of stable solutions and acausal propagation of perturbations. We will follow Calzetta [19] and use the framework of more causal and stable theories such as the Israel-Stewart 'second-order' type theory or the Geroch-Lindblom 'divergence type' description of a relativistic real fluid. A covariant theory has the following rules.

(a) Intensive quantities $(T, p, \mu)$ are associated with scalars, which represent the value of the quantity at a given event, as measured by an observer at rest with respect to the fluid.

(b) Conjugate extensive quantities $(S, V, N)$ are associated with vector currents $\left(S^{\alpha}, \hat{u}^{\alpha}, N^{\alpha}\right)$. If any of the extensive currents $X^{\alpha}$ is conserved, then $X_{; \alpha}^{\alpha}=0$. The quantity $\hat{u}^{\alpha}$ associated with the volume is the fluid unit 4-velocity.

(c) Energy and momentum are combined into a single extensive quantity and associated with the tensor $T^{\alpha \beta}$. The energy current is $U^{\alpha}=T^{\alpha \beta} \hat{u}_{\beta}$.

The entropy current $S^{\alpha}$ is given by

$$
T S^{\alpha}=T^{\alpha \beta} \hat{u}_{\beta}+p \hat{u}^{\alpha}-\mu N^{\alpha},
$$

which is rewritten as

$$
S^{\alpha}=T^{\alpha \beta} \beta_{\beta}+\Phi^{\alpha}-\alpha N^{\alpha}
$$

with affinity $\alpha=\mu / T$, thermodynamic potential $\Phi^{\alpha}=p \beta^{\alpha}$, and inverse temperature vector $\beta^{\alpha}=\hat{u}^{\alpha} / T . N^{\alpha}=n \hat{u}^{\alpha}$, where $n$ is the particle number density seen by a comoving observer.

Suppose the fluid departs from equilibrium by a fluctuation $\delta N^{\alpha}, \delta T^{\alpha \beta}$, consistent with the conservation laws but otherwise arbitrary. Then the change in entropy production is

$$
\delta S_{; \alpha}^{\alpha}=-\alpha_{, \alpha} \delta N^{\alpha}+\beta_{\beta ; \alpha} \delta T^{\alpha \beta} .
$$

For a true equilibrium state the entropy must be stationary [20], and so $\alpha_{, \alpha}=\beta_{(\beta ; \alpha)}=0$. Thus the affinity must be constant, and the inverse temperature vector must be Killing. 
If $\xi^{\mu}$ generates a timelike conformal symmetry then one has thermal equilibrium with inverse temperature $\beta^{\mu}=\xi^{\mu} / T$ as a conformal Killing vector for trace-free $\delta T^{\alpha \beta}$. In the exterior Vaidya region we have a weaker matter symmetry $\mathcal{L}_{\xi} g_{\alpha \beta}^{\text {Vad }}=-r R_{\alpha \beta}$ and so $\delta S_{; \alpha}^{\alpha} \neq 0$. The physical dimensions of $\dot{S}$ are energy/(s K) and, reasoning dimensionally,

$$
\frac{\dot{m}}{r}\left(\frac{k_{B} G}{c^{4}}\right) \sim \dot{S}
$$

in units with $k_{B}=G=c=1$. If one examines the terms of equation (22) its clear that $\Phi^{\alpha}$ and $N^{\alpha}$ lie along $\hat{u}^{\alpha} \partial_{\alpha}=A^{-1 / 2} \partial_{u} . T^{\alpha \beta} \beta_{\beta}$ has components along and orthogonal to $\hat{u}^{\alpha}$. We can thus write

$$
S^{\alpha}=S \hat{u}^{\alpha}+P^{\alpha}
$$

where $P^{\alpha} \hat{u}_{\alpha}=0$. The divergence of $S^{\alpha}$ is

$$
S_{; \alpha}^{\alpha}=S A^{-1 / 2}\left[\dot{S} / S+A^{-1}(\dot{m} / r)\right]+P_{; \alpha}^{\alpha}
$$

wherein the Vaidya luminosity gradient appears. The logarithm of $S$ measures the number of available states and we see here that $\dot{m} / r$ provides a measure of the rate of change in the number of states.

\section{Discussion}

Luminosity is one of the key observations made of astrophysical objects (a mass-luminosity relation is an important feature of main sequence stars). Use of the Bach current to compute luminosity gradients provides a covariant method for obtaining an astrophysically important quantity. We have identified the luminosity gradient with entropy production in the Vaidya exterior. The Vaidya exterior dissipates energy by sending 'short-wavelength photons' to null infinity. For an equilibrium ensemble of star models, it is the Bach current and the rate of entropy increase that allows use of the fluctuation-dissipation theorem to measure the rate at which an initial deviation from equilibrium decays.

A full development of dissipative thermodynamics using the Bach current requires analysing the collapsing Vaidya interior, which we leave for future work. The Vaidya spacetime is only a first approximation to interesting systems.

\section{Acknowledgments}

I am indebted to Josh Goldberg, Jean Krisch, and Ted Newman for reading and commenting on early versions of this work

\section{References}

[1] Szekeres P 1968 Proc. R. Soc. A 304113

[2] Kozameh C N, Newman E T and Tod P 1985 Gen. Rel. Grav. 17343

[3] Penrose R and Rindler W 1986 Spinors and Space-Time vol 2 (Cambridge: Cambridge University Press) p 127

[4] Querella L 1998 Variational principles and cosmological models in higher-order gravity Doctoral Dissertation Université de Liège

[5] Dzhunushaliev V and Schmidt H-J 2000 J. Math. Phys. 413007

[6] Komar A 1959 Phys. Rev. 113934

[7] Goldberg J N 1990 Phys. Rev. D 41410

[8] Glass E N 1993 Phys. Rev. D 47474

[9] Goldberg J N 1980 General Relativity and Gravitation vol 1, ed A Held (New York: Plenum) 
[10] Penrose R 1982 Proc. R. Soc. A 38153

[11] Brown J D, Lau S R and York J W 1999 Phys. Rev. D 59064028

[12] Nurowski P and Plebanski J F 2000 Class. Quantum Grav. 18341

[13] Synge J L 1957 Proc. R. Irish Acad. A 591

[14] Santos N O 1985 Mon. Not. R. Astron. Soc. 216403

[15] Glass E N 1990 J. Math. Phys. 311974

[16] Lindquist R W, Schwartz R A and Misner C W 1965 Phys. Rev. B 1371364

[17] Singh T P and Vaz C 2000 Phys. Lett. B 48174

[18] Glass E N 1999 Proc. 8th Canadian Conf. General Relativity and Relativistic Astrophysics (AIP Conf. Proc. 493) 77

[19] Calzetta E 1998 Class. Quantum Grav. 15653

[20] Israel W 1988 Covariant fluid mechanics and thermodynamics: an introduction Relativistic Fluid Dynamics ed A Anile and Y Choquet-Bruhat (New York: Springer) 